\title{
La actualidad del concepto sobrenaturaleza de José Ortega y Gasset: una mirada desde la inteligencia artificial ${ }^{*}$
}

\author{
Antonio Luis Terrones Rodríguez
}

Recibido: 20 de mayo de 2019 • Aprobado: 30 de septiembre de 2019

\section{Resumen}

Los primeros textos en los que José Ortega y Gasset reflexionaba sobre la técnica se publicaron en 1935. Ha pasado casi un siglo y este texto pretende rescatar uno de los conceptos que el filósofo español presentó en Meditación de la técnica, a saber, la sobrenaturaleza. En la actualidad, el gran reto que la tecnología se ha impuesto ha sido el de llevar a su máximo exponente a la inteligencia artificial, para hacerla un medio desafiante de los límites que nos impone la naturaleza. Entre las propuestas más destacadas dentro del campo de los sistemas artificiales se encuentra la superinteligencia y la singularidad, dos de los deseos más anhelados por pensadores como Nick Bostrom o Raymond Kurzweil. Así pues, si el campo tecnológico está centrando grandes esfuerzos en el desarrollo de la inteligencia artificial, deberíamos preguntarnos si la motivación que se encuentra detrás de tal impulso se fundamenta verdaderamente en la necesidad humana de una sobrenaturaleza de la que tanto nos hablaba Ortega y Gasset.

Palabras clave: inteligencia artificial, proyecto vital, singularidad, sobrenaturaleza, superinteligencia.

* Artículo de reflexión. Citar como: Terrones, A. (2020). La actualidad del concepto de sobrenaturaleza de José Ortega y Gasset: una mirada desde la inteligencia artificial. Análisis, 52 (96), 165-181. Dol: https://doi.org/10.15332/21459169/5129

** Docente de la Escuela de Filosofía de la Pontificia Universidad Católica del Ecuador, Quito (Ecuador). Estudiante de doctorado en la Universitat de Valencia (España). Dirección postal: Facultad de Filosofía y Ciencias de la Educación, Av. Blasco Ibáñez 30, 46010 Valencia, España. Correo electrónico: antonioluis.terrones@gmail.com. ORciD: http://orcid. org//0000-0002-4015-8537 


\section{The relevance of José Ortega y Gasset's supernatural concept: a look from artificial intelligence}

Antonio Luis Terrones Rodríguez

\section{Abstract}

The first texts in which José Ortega y Gasset reflected on the technique were published in 1935. It has been almost a century and this text intends to rescue one of the concepts that the Spanish philosopher presented in Meditation on technique, namely supernatural. At present, the great challenge that technology has imposed has been to take its maximum exponent to artificial intelligence, to make it a challenging means of the limits that nature imposes on us. Among the most outstanding proposals within the field of artificial systems is superintelligence and uniqueness, two of the desires most longed for by thinkers such as Nick Bostrom or Raymond Kurzweil. So, if the technological field is focusing great efforts on the development of artificial intelligence, we should ask ourselves if the motivation behind such an impulse is truly based on the human need for a supernatural that Ortega y Gasset talked about so much.

Keywords: artificial intelligence, vital project, uniqueness, supernatural, superintelligence. 


\section{Actualité du concept de surnature de José Ortega y Gasset : un regard à partir de l'intelligence artificielle}

Antonio Luis Terrones Rodríguez

\section{Résumé}

Les premiers textes contenant des réflexions de José Ortega y Gasset sur la technique datent de 1935. Après presque un siècle, ce texte se propose de revenir sur l'un des concepts traités par le philosophe espagnol dans Meditación de la técnica, à savoir la surnature. Aujourd'hui, le grand défi de la technologie consiste à mener au bout les possibilités propres à l'intelligence artificielle, afin qu'elle devienne un milieu qui étende les limites imposées par la nature. Par ailleurs, les systèmes artificielles cherchent à accomplir la super-intelligence et la singularité, tel que le désiraient Nick Bostrom ou Raymond Kurzweil. Cela veut dire que la technologie s'efforce de plus en plus à développer l'intelligence artificielle, ce qui pose la question de savoir si la motivation de tels efforts se fonde dans le besoin humain de la surnature dont parle José Ortega y Gasset.

Mots clés: intelligence artificielle, projet vital, singularité, surnature, super-intelligence. 
Es, pues, la técnica, la reacción enérgica contra la naturaleza

Ortega y Gasset (1965b, p. 21).

\section{Introducción}

Según Diéguez (2017), la reflexión sobre la técnica del filósofo José Ortega y Gasset, y concretamente sobre su obra Meditación de la técnica (1965b), fuente de su filosofía de la tecnología, ha sido escasamente tratada, pese a su carácter vanguardista. Algunos intentos de la Revista de Occidente en el 2000 y de la Fundación Ortega-Marañón, con la organización de un congreso internacional sobre la técnica en Ortega, demuestran un claro interés por recuperar el pensamiento orteguiano para interpretarlo a la luz del presente. Así pues, el objetivo principal de este trabajo consiste en rescatar uno de los conceptos más importantes de la filosofía de la técnica del filósofo español, la sobrenaturaleza, para llevar a cabo un ejercicio de contextualización desde la óptica de la inteligencia artificial (IA). Es importante recoger el testigo de pensadores que, como Ortega, han contribuido a un mejor conocimiento de la condición técnica del ser humano, y ponerlo en valor desde el presente.

En el relato orteguiano subyacen tres caracteres antropológicos que resultan fundamentales para entender la dimensión técnica del ser humano: ingeniosidad, carácter constructivo y superioridad natural (Gehlen, 1993). Estas características antropológicas le sirven al ser humano para plantear su vida como proyecto y para poder desplegar sus deseos de bienestar en el mundo, un bienestar que es ilimitadamente variable y que responde a las necesidades del momento. La técnica aquí se entiende como una reacción enérgica capaz de desafiar la naturaleza y superar los límites que esta le impone al humano.

Actualmente, la sobrenaturaleza se encuentra implícita en los sueños creadores de sistemas artificiales que sean capaces de superar las capacidades que configuran la inteligencia del ser humano. En ese sentido, se llevará a cabo el establecimiento de un vínculo entre el concepto de sobreanaturaleza orteguiano, que será expuesto en la primera parte del trabajo, y dos postulados del ámbito de la IA, superinteligencia y singularidad. El planteamiento de este vínculo responde a la necesidad de observar desde la óptica actual el pensamiento de Ortega y destacar la importancia que tiene la IA para la vida, entendida como un proyecto tecnológico que forma parte de la histórica condición técnica del ser humano en su empeño por responder enérgicamente a las limitaciones impuestas por la naturaleza.

\section{La técnica como recurso para la sobrenaturaleza}

Mitcham (1989) reconoce que José Ortega y Gasset es el primer filósofo en ocuparse de la cuestión tecnológica. La obra más importante del filósofo español 
donde se aborda el tema de la técnica es Meditación de la técnica (1965b). En el prólogo, Ortega señala que esa obra nace a partir de un curso que fue desarrollado en 1933 en la Universidad de Verano de Santander. Sin embargo, posteriormente a ese curso, las lecciones dictadas fueron fragmentadas y publicadas en artículos dominicales en el periódico La Nación de Buenos Aires, en 1935. Finalmente, Meditación de la técnica fue publicada en 1939, junto con otro ensayo: "Ensimismamiento y alteración". También existe otro importante texto que recoge la conferencia El mito del hombre allende la técnica (1965a), pronunciada por Ortega en 1951 en la ciudad alemana de Darmstadt. Como señala Esquirol (2011), la reflexión de Ortega sobre la técnica se "inserta en el núcleo de su filosofía de la vida y de su comprensión de la condición humana" (p. 15). Hay una obra muy importante en la historia del pensamiento de Ortega, anterior a su Meditación de la técnica, y que marcará en su obra un antes y un después, $L a$ rebelión de las masas, de 1929. En esta obra se identifica la técnica como la generadora del hombre masa. Aunque los tratamientos que recibe la técnica en las dos últimas obras mencionadas son diferentes, coinciden al proporcionar unas orientaciones fundamentales para poder comprender la influencia que tiene dicha técnica sobre el ser humano y su vida. La técnica se encuentra en una posición relacional con la existencia, articulándose a partir de tres conceptos claves: necesidad, extrañamiento y proyecto.

El humano vive inmerso en circunstancias, rodeado de la naturaleza que le impone unas necesidades que debe satisfacer, como protegerse del frío o comer. La vida se encuentra ligada a la necesidad y el humano se empeña por cubrir esas necesidades porque quiere vivir. En ese sentido, se pone en marcha una serie de actividades para esperar satisfacer las necesidades, estableciendo así las condiciones indispensables para que puedan satisfacerse, y crea, por ejemplo, sistemas de cultivo, habitáculos con los que poder resguardarse del frío, etc. Este tipo de actividades significan la puesta en suspenso de las necesidades más primarias, como señala el propio filósofo, "calefacción, agricultura y fabricación de carros o automóviles no son, pues, actos en que satisfacemos nuestras necesidades, sino que, por el pronto, implican lo contrario: una suspensión de aquel repertorio primitivo de haceres en que directamente procuramos satisfacerlas" (Ortega y Gasset, 1965b, p. 18).

A partir de esta primera consideración sobre la necesidad, surge la primera definición que Ortega hace en su obra:

Es la técnica, que podemos, desde luego, definir como la reforma que el hombre impone a la naturaleza en vista de la satisfacción de sus necesidades [...] Es, pues, la técnica, la reacción enérgica contra la naturaleza o circunstancia que lleva a crear entre ésta y el hombre una nueva naturaleza puesta sobre aquélla, una sobrenaturaleza. Conste, pues: la técnica no es lo que el hombre hace para satisfacer sus necesidades [...] La técnica es la reforma de la naturaleza, de esa naturaleza que nos hace necesitados y menesterosos, reforma en sentido tal que las necesidades quedan, a ser posible, anuladas por dejar de ser problema su satisfacción. (1965b, pp. 21-22) 
El ser humano no se adapta a las circunstancias que le vienen dadas y, por eso, reacciona ante estas para estar bien, de manera que no se resigna, porque no se trata únicamente de "estar" en el mundo, sino de "estar bien". Por eso, se las ingenia para construir la sobrenaturaleza sobre la que reflexiona el filósofo español. Pero el verdadero empeño consiste en estar bien en el mundo, por lo que reúne todas sus fuerzas para garantizar ese bienestar. Lo objetivamente superfluo se convierte, entonces, en lo que es visto como únicamente necesario (1965b). Esto que Ortega sostiene sobre las necesidades biológicas, objetivamente hablando, no son necesidades como tal para el humano, sino que más bien se convierten en verdaderas necesidades cuando condicionan su estar en el mundo, que en ese sentido son necesidades consideradas subjetivas. Ortega sostiene qe son subjetivas, porque ese estar en el mundo es lo que garantiza el posterior bienestar y lo que es aceptado como superfluo.

Recordando el concepto de extrañamiento que ha sido mencionado anteriormente, es improtante mencionar que el humano crea un mundo diferente al anterior mundo que le es dado, porque no se siente perteneciente a ese mundo; se siente extraño en el mismo, no se siente cómodo. El humano se encuentra ante un mundo que le presenta circunstancias que hacen que no se sienta identificado, un mundo que le es extraño; por eso, surge en él la voluntad, el empeño de construir un nuevo mundo, una nueva naturaleza, una sobrenaturaleza en la que se vea reflejado y que le aleje del extrañamiento. El mundo dado es extraño, razón por la cual es creado otro a partir de la técnica y del impulso por una sobrenaturaleza. Esquirol (2011) realiza una importante aportación cuando señala que la idea de extrañamiento es similar a la de ser "arrojado" de Heidegger

El concepto de necesidad se encuentra vinculado al de bienestar, aunque resulta difícil determinar en qué consiste el bienestar, pues en materia de bienestar y técnica parece ser que no existe acuerdo. El bienestar es relativo al tiempo, al espacio, a la cultura; por lo tanto, varía con el tiempo y con las gentes, motivo por el cual se torna complejo delimitar su conceptualización. Así pues, el concepto de bienestar es variable, ya que se encuentra vinculado a una idea filosófica de cómo es entendida la vida. Esto quiere decir que, en función del proyecto de vida que se decide perseguir, la idea de lo que se entiende como bienestar tendrá que adaptarse a las exigencias y necesidades.

Pero también es importante mencionar que el carácter cambiante del bienestar va acompañado del cambio de técnica y aquí es donde es necesario rescatar unas palabras de Ortega (1965b):

Basta con que cambie un poco sustancialmente el perfil del bienestar que se cierne sobre el hombre, que sufra una mutación de algún calibre la idea de la vida, de la cual, desde la cual y para la cual

1 Se recomienda llevar a cabo un acercamiento al concepto de la técnica que Heidegger (1997) esboza en La pregunta por la técnica. Aunque su postulado es posterior al de Ortega y no versa estrictamente sobre su concepto de sobrenaturaleza, puede servir para enriquecer la reflexión. 
hace el hombre todo lo que hace, para que la técnica tradicional cruja, se descoyunte y tome otros rumbos. (p. 32)

En este sentido, la técnica es también cambiante, como señala el filósofo español, pues depende de la idea de bienestar.

La técnica genera un vacío, ya que trata de ahorrar esfuerzos. Piénsese, por ejemplo, en el mundo actual, donde surgen artefactos tecnológicos que sirven para la liberación de tareas, como por ejemplo las zapatillas inteligentes, los sensores para el jardín, las puertas de garaje inteligentes, los wearables para perros, los reguladores de la luz, el cubo de basura inteligente, etc. Se quieren satisfacer las necesidades y estar bien con el mínimo esfuerzo. En resumen, los actos técnicos no consisten en llevar a cabo un esfuerzo para satisfacer de forma directa las necesidades, ya sean objetivas o subjetivas (superfluas), sino que son aquellos en los que se lleva a cabo una reacción frente a las circunstancias que requieren de esfuerzo, en primer lugar para inventar y, posteriormente, para ejecutar un plan o proyecto que ha sido definido previamente. Según Ortega (1965b), ese plan o proyecto al que se hace referencia debe permitir:

1. Asegurar la satisfacción de las necesidades, por lo pronto, elementales.

2. Lograr esa satisfacción con el mínimo esfuerzo.

3. Crearnos posibilidades completamente nuevas produciendo objetos que no hay en la naturaleza del hombre. Así, el navegar, el volar, el hablar con el antípoda mediante el telégrafo o la radiocomunicación.

El ser humano consigue enfrentarse a las circunstancias y desafiarlas por medio de la reforma que brinda la técnica, consiguiendo de ese modo reducir el esfuerzo impuesto por tal circunstancia, que en definitiva es dominada para la creación de una sobrenaturaleza. Además, en esa acción técnica, caracterizada por el ahorro de esfuerzo, también se encuentra la búsqueda de seguridad, pues las circunstancias conducen a un espacio de incertidumbre e inseguridad que dificulta el pleno desenvolvimiento y provoca extrañamiento.

Ortega (1965b) alerta de la decadencia cultural a la que conduce el progresismo basado en la fe ciega e irreflexiva en la técnica. La falta de reflexividad provoca una confusión en la humanidad, pues se identifica la sobrenaturaleza con la naturaleza, perdiendo enteramente la conciencia de la técnica que está siendo utilizada. Este constante predominio de la técnica en la vida ha generado una dificultad al momento de poder vivir materialmente sin ella.

\section{La vida como proyecto}

Han sido abordados los conceptos de necesidad y extrañamiento para poder llevar a cabo un acercamiento con claridad al pensamiento de Ortega sobre la 
técnica, pero aún queda reflexionar sobre el concepto de proyecto, que enlaza con su propuesta de razón vital e histórica. El ahorro de esfuerzo que promueve la técnica crea posibilidades para emplear el tiempo e imaginar proyectos. Como la técnica libera de esfuerzo, ¿a qué se dedica el tiempo libre? Según el filósofo español, ahí es donde el ser humano debe inventar su vida, hacerla él mismo, como si fuera un "artesano de su propia vida", expresión que utiliza Cortina (2013). De ese modo, el humano tendría que crear el propio relato de su vida, tendría que proyectarse a sí mismo. En ese sentido, la técnica se encontraría ligada al concepto de lo que significa ser humano, pues adquiriría un carácter antropológico y ontológico. La filosofía de la técnica de Ortega se construye sobre su idea de la vida humana, entendida esta como un fenómeno que da forma a su significado en la relación activa con las circunstancias, es decir, como un activo creador de esas circunstancias. Es un proyecto de vida que forja su razón de ser en la interacción entre el humano y las circunstancias.

La vida humana no es algo que esté dado por naturaleza, algo que se encuentre determinado enteramente por dicha naturaleza; por tanto, no está dada por la existencia. Al contrario, la persona tiene que crearse a sí misma elaborando un proyecto de vida. El humano desliza su existencia desde una actividad autointerpretativa y autocreativa, poniendo el acento en él mismo, pero también en las circunstancias que lo motivan a reaccionar proyectando. En esta existencia activa se halla una imaginación creativa que pone su poder a disposición del proyecto personal que quiere realizarse. Una vez que el humano ha decidido qué proyecto asume y emprende, son necesarios los recursos materiales, técnicos, para poder realizar tal proyecto. En este sentido, para Ortega la técnica supone una apertura de nuevas posibilidades orientadas a hacer la vida, a hacer esa vida en la que el humano escribe su relato porque es proyecto, ya sea gentleman, bodhisattva o hidalgo. La vida del humano no está definida por naturaleza, está abocada a ser un proyecto que está por realizarse y es producto de su imaginación creativa.

A partir de los sugerido acerca de que el humano se hace a sí mismo, en la línea del pensamiento de Ortega podría ser identificado como un homo faber, pero no como un faber que se limita únicamente a la producción material, sino también como un faber que se encarga de autoproyectarse y de escribir el relato de su propia existencia. El filósofo español está aplicando el esquema técnico al hacerse del humano entendido como proyecto, poniendo el acento sobre la idea de construcción; por eso, utiliza la expresión "autofabricarse", "de ahí que nuestra vida sea pura tarea e inexorable quehacer" (Ortega y Gasset, 1965b, p. 51).

La forma que Ortega presenta de entender la vida se fundamenta en su modo de entender la razón que está en profunda conexión con la experiencia de la vida, pues a partir de ella se nutre. Su idea de la vida fue expuesta en el Discurso para los Juegos Florales de Valladolid de 1906, y en ella entiende el vivir como "más vivir", como aumento del propio ser o "henchimiento", como diría Conill (2016, p. 75). La postura de Ortega acerca de la vida está inspirada en el pensamiento de Nietzsche. En este sentido, como la vida es adaptación a las circunstancias, es también creación, es atrevimiento y voluntad vital. 
Como acaba de mencionarse, el modo de entender la vida que tiene Ortega se elabora desde su nueva filosofía de la razón vital, que en cierta medida recoge el testigo de varios aspectos del pensamiento nietzscheano. Además, la aportación de Ortega se centra principalmente en su reflexión acerca de la crisis de los deseos y la necesidad que plantea a la hora de cultivar y forjar los proyectos de vida, pues los deseos tecnológicos marginan el verdadero deseo del humano, el deseo de ser sí mismo, y desplaza la preocupación por un proyecto personal. Así pues, en la crisis de los deseos orteguiana se hace hincapié en que los deseos superfluos alimentan el vacío interior. El ser humano se encuentra desconcertado, saturado ante tanta tecnología, y en cierto sentido alimenta su deseo artefactual. Sin embargo, se siente inquieto por la conciencia de su principal ilimitación, una ilimitación ante el superávit de posibilidades inherentes en la tecnología.

\section{La inteligencia artificial y su actualidad}

Debido a que el objeto de la reflexión de estas páginas se encuentra orientado a la reflexión sobre la actualidad del concepto de sobrenaturaleza de Ortega a partir de la IA, es importante llevar a cabo una breve introducción a esta asombrosa y novedosa artillería tecnológica. Es fundamental comenzar la tarea de esclarecimiento tratando de delimitar el concepto y significado de IA, con el fin de facilitar la comprensión sobre el fenómeno de los sistemas artificiales.

Como ocurre en infinidad de ámbitos en lo que se refiere a la conceptualización, existen muchas definiciones en torno a la IA, cada una propuesta desde diferentes enfoques, aunque parece ser que todas tienen un punto en común. Ese punto en común consiste en una idea fundamental en torno a la que giran las diversas propuestas, a saber, la idea de crear y dar forma a programas de ordenador o también a máquinas, que sean capaces de desarrollar conductas que serían consideradas inteligentes si las realizara un ser humano. Esta definición es abierta y puede generar consenso, pues la variedad de definiciones facilitadas por algunos expertos en la materia suelen ser cerradas y diferenciarse unas de otras, por lo que, al menos en este caso, es más prudente no cerrarse. Esta definición que se fundamenta en la búsqueda de la emulación del cerebro humano es similar a la propuesta formulada por John McCarthy, Marvin L. Minsky, Nathaniel Rochester y Claude E. Shannon en 1955 (Copeland, 1996). Además, en lo relativo a la definición de ıA, la británica Boden (2017) también señala lo siguiente:

La inteligencia artificial (IA) tiene por objeto que los ordenadores hagan la misma clase de cosas que puede hacer la mente.

Algunas (como razonar) se suelen describir como "inteligentes". Otras (como la visión), no. Pero todas entrañan competencias psicológicas (como la percepción, la asociación, la predicción, la planificación, el control motor) que permiten a los seres humanos y demás animales alcanzar sus objetivos. 
La inteligencia no es una dimensión única, sino un espacio profusamente estructurado de capacidades diversas para procesar la información. Del mismo modo, la IA utiliza muchas técnicas diferentes para resolver una gran variedad de tareas.

[...] La IA tiene dos objetivos principales. Uno es tecnológico: usar los ordenadores para hacer cosas útiles (a veces empleando métodos muy distintos a los de la mente). El otro es científico: usar conceptos y modelos de IA que ayuden a resolver cuestiones sobre los seres humanos y demás seres vivos. (Boden, 2017, pp. 11-12)

Lo expuesto en el párrafo anterior no niega la posibilidad de que puedan existir consensos en torno a ciertos marcadores de la inteligencia en numerosos contextos concretos. La duda surge a la hora de intentar aplicar esos marcadores a la máquina. Por ejemplo, si se tiene en cuenta la ardua tarea realizada por los escribas en el antiguo Egipto con los manuscritos para reproducir textos, con el fin de transmitir los conocimientos, y se la compara con una imprenta de libros de textos escolares de la actualidad, la máquina sería "más inteligente" porque es más rápida reproduciendo textos que un ser humano; en este caso, ha sido tenido en cuenta el marcador de la velocidad. Como puede comprobarse, el marcador de la velocidad no es un indicador válido para considerar a una máquina más inteligente que un ser humano.

Considerar las capacidades humanas como un criterio válido para valorar la iA puede suponer un problema. Una máquina puede llegar a realizar una tarea en milisegundos, y una persona no sería capaz de realizar una tarea parecida en ese corto periodo; por eso, podríamos llegar a pensar que la máquina parece dar muestras de inteligencia. Episodios de ese tipo van a ocurrir en las próximas décadas en cientos de ámbitos, incluso en muchos ya se han producido, por lo que la utilización del método comparativo entre la inteligencia humana y la iA es algo que puede conducir al absurdo, pues la inteligencia humana siempre saldría perdiendo en diversidad de situaciones. Esto muestra una vez más que la tarea de tratar de ofrecer una definición concreta de la iA no es nada fácil.

En los últimos años viene hablándose de la cuarta revolución industrial. Schwab (2016), por ejemplo, dedica sus esfuerzos en una de sus obras a analizar cómo la IA está jugando un importante papel en la nueva revolución tecnológica que está transformando la humanidad por medio de la convergencia entre sistemas digitales, físicos y biológicos. Las nuevas tecnologías más avanzadas que se están promoviendo desde la iA provocan cambios drásticos en la forma en cómo se establece la relación con el mundo, el trabajo y la organización de la vida. Rouhiainen (2018), por su parte, considera que en la cuarta revolución la iA es el elemento más importante, debido a que representa un importante eje articulador y cohesionador de las demás partes componentes (p. 38). El desafío de la cuarta revolución industrial se encuentra en saber afrontar una serie de cambios que son fruto del crecimiento exponencial para los que la ciudadanía no está preparada. La humanidad se enfrenta a un doble desafío en el presente de la IA, a saber, entender bien estas tecnologías y también a saber cómo usarlas bien. 
Una vez que han sido expuestas algunas de las principales características de la IA y su aplicación actual, es importante pasar a esbozar algunas de las propuestas más destacadas de los últimos años en torno al futuro y desarrollo de los intelectos sintéticos que puede girar en torno a una actualización de concepto de sobrenaturaleza de Ortega y Gasset: superinteligencia y singularidad.

\section{Superinteligencia y singularidad}

Bostrom (2016) define la superinteligencia como "cualquier intelecto que exceda en gran medida el desempeño cognitivo de los humanos en prácticamente todas las áreas de interés" (p. 22). El filósofo sueco destaca por ser un férreo defensor del movimiento transhumanista y, por lo tanto, defiende la idea sobre la existencia de una mayor facilidad a la hora de desarrollar la inteligencia en una base artificial antes que en una base biológica, ya que las máquinas tienen una serie de ventajas que las entidades biológicas no tienen.

No es nueva la idea de la superación de niveles humanos. Por ejemplo, los gatos tienen un olfato más fino que los humanos, y una calculadora realiza ejercicios matemáticos mucho más rápido que un profesor de matemáticas de carne y hueso. No obstante, cuando se habla de intelectos artificiales, subyace una serie de entidades añadidas que tienen una inteligencia de tal magnitud que pueden ser capaces de sustituir a los seres humanos en cualquiera de sus ámbitos de desempeño. Así, Bostrom (2016) propone la siguiente clasificación para diferenciar las superinteligencias: superinteligencia de velocidad, superinteligencia colectiva y superinteligencia de calidad.

Bostrom (2016) considera que cualquiera de los tres tipos de superinteligencia podría llegar a ser capaz de desarrollar alguna de las demás. En este sentido, pueden suponerse, pues existen motivos para ello, y es muy probable que una vez que la is alcance los niveles humanos de inteligencia se produzca una explosión de superinteligencia, lo que implicaría que los intelectos sintéticos fueran autónomos al margen de los programadores, y que pudieran constituir y dar forma a su vez a otros intelectos. Por lo tanto, el fenómeno de la superinteligencia invita a una profunda reflexión ética, pues no se está hablando de un tema baladí, sino de un tema que compromete de una manera importante a la humanidad.

La denominada "cinética" de una explosión de inteligencia por parte de Bostrom (2016) muestra cómo podría tener lugar la sincronización y la velocidad de despegue en el momento en el que la iA alcanzara niveles cognitivos humanos. Llegado el momento en el que la is alcanzara los mismos niveles cognitivos que los humanos, existen diferentes caminos que pueden divisarse en el horizonte. Sin embargo, y atendiendo a la reflexión que suscita este texto de Bostrom (2016), es importante partir de la consideración del fenómeno de la transición, debido a la existencia de una supuesta gran probabilidad de explosión de superinteligencia. Aunque esta consideración parte de un estudio predictivo, 
no debería menospreciarse la importancia del estudio realizado por Müller y Bostrom (2014).

Tabla 1. Resultados de encuestas a expertos en IA sobre cuándo esperan que surja una inteligencia artificial de nivel humano

\begin{tabular}{|l|c|c|c|}
\hline \multicolumn{4}{|c|}{ ¿Cuándo conseguiremos una inteligencia artificial de nivel humano? } \\
\hline & $\mathbf{1 0} \%$ & $\mathbf{5 0} \%$ & $\mathbf{9 0} \%$ \\
\hline PT-AI & 2023 & 2048 & 2080 \\
\hline AGI & 2022 & 2040 & 2065 \\
\hline EETN & 2020 & 2050 & 2093 \\
\hline TOP 100 & 2024 & 2050 & 2070 \\
\hline Combinados & 2022 & 2040 & 2075 \\
\hline
\end{tabular}

Fuente: Adaptada de Bostrom (2016, p. 20).

En la tabla 1 se muestran los resultados de cuatro encuestas diferentes, así como la combinación de estos. Los participantes de las encuestas se reflejan en el documento de Müller y Bostrom (2014). A pesar del carácter predictivo de las encuestas, Bostrom es cercano a la idea que defiende la posibilidad del surgimiento de la superinteligencia poco después del momento en el que la iA alcance un nivel humano. Bostrom (2016) no es el único en sostener esta idea, ya que existen otras figuras reconocidas como Barrat (2015) o Tegmark (2017) que también se sitúan en su estela.

Singularidad es otro término que se utiliza en el campo de la is para referirse al sistema supertinteligente que es capaz de perfeccionarse a sí mismo y crear otros sistemas, incluso más inteligentes que él, siguiendo un crecimiento exponencial. Aquí se podría mencionar brevemente la cuestión de la cinética de la explosión de superinteligencia, o de la singularidad, pues está muy vinculada al ritmo de crecimiento. Los defensores de la singularidad afirman que se dará lugar cuando en un futuro los mejores desarrolladores no sean personas de carne y hueso, sino propiamente IA, el rendimiento de la IA, que en un primer momento se atribuirá al hardware y posteriormente, tras un revisionismo, al software, se duplicará considerablemente, y la velocidad infinita será la norma habitual. Por lo tanto, si la velocidad con la que la ia va a diseñarse en el futuro por sí misma va a ser infinita, entonces el nivel superior de inteligencia es muy probable y casi evidente, lo que podría hacer pensar que la explosión de superinteligencia será posible.

El máximo exponente de la singularidad es el estadounidense Raymond Kurzweil (2017), quien considera que la singularidad puede perfeccionarse a sí misma, teniendo como horizonte la constitución de todo un universo basado en una entidad global inteligente. El estadounidense afirma que, cuando un intelecto sintético supere a la inteligencia humana, el progreso será mucho más rápido. Kurzweil (2017), al igual que Moravec (1988), está convencido de que 
durante la primera mitad del siglo xxi las máquinas excederán la inteligencia humana. Según este especialista de la IA, el crecimiento de la iA será exponencial:

\begin{abstract}
Representa la fase casi vertical del crecimiento exponencial que tiene lugar cuando el ritmo es tan extremadamente alto que la tecnología parece expandirse a una velocidad infinita, pese a que, desde la perspectiva matemática, no hay discontinuidad ni ruptura y los ritmos de crecimiento siguen siendo finitos, aunque extraordinariamente grandes. Pero desde nuestro limitado marco actual este evento inminente parece una ruptura aguda y brusca en la continuidad del progreso. (Kurzweil, 2017, p. 26)
\end{abstract}

La singularidad está cerca, como reza el título de la obra de Kurzweil (2017), y va a implicar un cambio de paradigma en varios campos que este especialista norteamericano menciona en su obra. A finales de este siglo, se espera que la mayor parte de la inteligencia sea no biológica; sin embargo, eso no supone el fin de la inteligencia biológica. Kurzweil (2017) es un férreo defensor de la singularidad y, en este sentido, es muy optimista, pues considera que las inteligencias sobrehumanas servirán para satisfacer nuestras necesidades y deseos. Su propuesta para evitar la anulación humana, ya que es muy probable que el ser humano quede a merced de las máquinas o, como Carr (2014) sostiene, "atrapados", es la fusión con la máquina, aunque él se refiere a este aspecto como "enlace íntimo" (Kurzweil, 2017, p. 33).

Las propuestas de Bostrom (2016) y de Kurzweil (2017) tienen mucho en común: mientras que el primero habla de superinteligencia, el segundo habla de singularidad. Ambos pensadores están convencidos de que la superinteligencia artificial llegará durante este siglo, influyendo en numerosos ámbitos humanos hasta su dominio, a no ser que se tomen medidas como las que proponen. De este modo, se propone, en el caso de Kurzweil (2017), una fusión íntima con la máquina y, en el caso de Bostrom (2016), la inserción de comportamientos éticos con contenido axiológico.

\title{
Los intelectos sintéticos como medio de superación de límites
}

Para Ortega y Gasset (1965b) la técnica representa un medio condicional al que el ser humano da forma y se adueña para poder desafiar los límites que la naturaleza le impone. La condición técnica que el filósofo español le confiere al ser humano puede establecer un estrecho vínculo, a modo de aclaración, con el concepto de homo faber que Arendt (2001) formula. Es conocida la distinción que Arendt (2001) establece en La condición humana entre labor, trabajo y acción, como aquellas actividades en las que el ser humano ha desplegado su vida: la labor, vinculada al proceso biológico del ser humano, teniendo por condición la vida misma; el trabajo, vinculado a las actividades de producción artificial de objetos; y la acción, que es la actividad que realizan los humanos entre sí sin 
la mediación de las cosas, teniendo la pluralidad como una condición propia. Mientras la labor y el trabajo se desarrollan en la esfera privada, la acción pertenece al ámbito público. Arendt (2001) caracteriza el trabajo en contraposición a la labor, tomando en cuenta la diferencia que Locke (2006) establece entre ambos conceptos: "la labor de nuestro cuerpo y el trabajo de nuestras manos" (p. 226). La autora señala lo siguiente:

Labor es la actividad correspondiente al proceso biológico del cuerpo humano, cuyo espontáneo crecimiento, metabolismo y decadencia final están ligados a las necesidades vitales producidas y alimentadas por la labor en el proceso de la vida. La condición humana de la labor es la misma vida. Trabajo es la actividad que corresponde a lo no natural de la exigencia del hombre, que no está inmerso en el constantemente repetido ciclo vital de la especie, $\mathrm{ni}$ cuya mortalidad queda compensada por dicho ciclo. El trabajo proporciona un «artificial» mundo de cosas, claramente distintas de todas las circunstancias naturales. Dentro de sus límites se alberga cada una de las vidas individuales, mientras que este mundo sobrevive y trasciende a todas ellas. La condición humana del trabajo es la mundanidad. (Arendt, 2012, p. 21)

Por medio del trabajo, señala Arendt (2012), los seres humanos producen objetos que plasman en su cultura y que tienen cierta durabilidad. Así pues, se distingue entre el animal laborans, que dedica sus esfuerzos a la subsistencia, y el homo faber, que crea un mundo que los seres humanos comparten entre sí. El homo faber desarrolla su producción mediante la evaluación, elección y empleo de los medios adecuados para alcanzar determinados fines. Además, la relación que el homo faber mantiene con el medio es la de adueñamiento y uso de la naturaleza; por ello, además, posee la capacidad de crear y destruir sus obras de consumo.

Para la antropología orteguiana, el ser humano es un proyecto en sí mismo, que centra sus esfuerzos en la escritura de su propio relato de vida. En ese sentido, la is representa un medio técnico para desafiar los límites que la naturaleza le impone al humano y, de ese modo, poder llevar a cabo la proyección de su sueño. La ia es un mecanismo técnico de desafío de la naturaleza limitante, es la creación de capacidades en las máquinas para hacer aquello que el ser humano no puede hacer debido a sus limitaciones naturales. El humano proyecta en los intelectos sintéticos aquello que él no puede ser, crea artificialmente capacidades para ir más allá de la naturaleza y situarse en una sobrenaturaleza. Esta creación de capacidades artificiales la lleva a cabo porque se autoimpone un patrón humano de bienestar que es ilimitablemente variable. Es el poder de la creación el que le otorga la posibilidad de caminar hacia una sobrenaturaleza ilimitada.

La iA es la máxima expresión en la actualidad de ese espíritu humano de infinita variabilidad que desafía constantemente los límites naturales. Es el resultado de un sueño que comenzó con Alan Turing (1950) y que llega hasta nuestros días con los Android Dreams de Toby Walsh (2017). A pesar del escepticismo y 
el temor con el que a veces se habla de la iA, esta es una de las grandes aventuras de nuestro tiempo. Los intelectos sintéticos tienen por objeto que los ordenadores hagan la misma clase de cosas que los seres humanos pueden hacer por medio de la capacidad intelectiva que se deriva del sistema cerebral; sitúan en un horizonte de posibilidades que hasta ahora solo era motivo de los relatos de ciencia ficción. La iA puede concebir múltiples aspectos de la realidad que el cerebro del ser humano es incapaz dadas sus limitaciones cognitivas naturales. Además, puede sobrepasar las fronteras biológicas de la memoria y acumular más información que un cerebro humano, así como procesar una mayor cantidad de datos a una velocidad que es imposible para los humanos. Estas son muestras de una superación de los mencionados límites que impone la naturaleza al ser humano y un modo de desafiarlas por medio de la técnica, en este caso representada por la IA.

\section{Conclusión}

El fortalecimiento del acceso más cercano a la dimensión técnica del ser humano es fundamental para la adquisición de un mayor conocimiento en términos antropológicos. Conceptos como el de sobrenaturaleza contribuyen positivamente a un cultivo del ejercicio de la comprensión sobre el humano y sus límites. Así pues, la reflexión de Ortega y Gasset (1965b) sobre la condición técnica ofrece un considerable enriquecimiento para la tarea filosófica.

Como ha podido observarse, el postulado del filósofo español encarna una importante vigencia debido al incuestionable reconocimiento de la dimensión técnica que configura la existencia humana. Esta existencia es entendida como proyecto y se encuentra estrechamente ligada a la condición técnica. Eso implica que la vida es entendida como proyecto a partir de las posibilidades que brinda la técnica y, en ese sentido, se orientan las acciones hacia la superación de los límites que la naturaleza impone como parte del proyecto que se ha decidido emprender.

Las diversas manifestaciones en las que la iA despliega su poder (técnologías de mejoramiento de la especie, nanorrobótica, vehículos autónomos, drones, etc.) representan una clara muestra del deseo constante por superar los límites que la naturaleza impone en sus diversas manifestaciones. Esta tecnología avanzada abre un abanico de posibilidades nunca antes conocidas para la humanidad, y esa apertura exige un mayor conocimiento sobre el ser humano. La pregunta por la sobrenaturaleza implíticita en la iA es también la pregunta por quién desea ser el humano, por el proyecto que se ha planteado llevar a cabo. Además, contribuye con un conocimiento de mayor profundidad sobre el sentido que es proyectado en el relato de la existencia y a la vez favorece la disposición de un mejor alcance de las limitaciones. La sobrenaturaleza es fruto del deseo por estar bien en el mundo, y eso exige pensar qué concepción del bienestar impulsa el diseño de las tecnologías actuales. 


\section{$\mathbf{R}_{\text {eferencias }}$}

Arendt, H. (2012). La condición humana. Barcelona: Paidós.

Barrat, J. (2015). Our final version. Artificial Intelligence and the End of the Human Era. New York: Thomas Dunne Books.

Boden, M. A. (2017). Inteligencia Artificial. Madrid: Turner Noema.

Bostrom, N. (2016). Superinteligencia, caminos, peligros, estrategias. Madrid: Teell Editorial.

Carr, N. (2014). Atrapados por las máquinas. Cómo las máquinas se apoderan de nuestras vidas. Madrid: Alfaguara.

Conill, J. (2016). De la razón pura a la razón vital orteguiana a través de Nietzsche. Revista de Hispanismo Filosófico, 21, 71-92.

Copeland, J. (1996). Inteligencia artificial. Madrid: Alianza Editorial.

Cortina, A. (2013). La ética. Barcelona: Paidós.

Diéguez, A. (2017). Transhumanismo. La búsqueda tecnológica del mejoramiento humano. Barcelona: Herder.

Esquirol, J. (2011). Los filósofos contemporáneos y la técnica: de Ortega a Sloterdijk. Barcelona: Gedisa.

Gehlen, A. (1993). Antropología filosófica. Del encuentro y descubrimiento del hombre por sí mismo. Barcelona: Paidós.

Heidegger, M. (1997). Filosofía, ciencia y técnica. Santiago de Chile: Editorial Universitaria.

Kurzweil, R. (2017). La singularidad está cerca. Cuando los seres humanos trascendamos la biología. Berlín: Lola Books.

Locke, J. (2006). Segundo tratado sobre el gobierno civil. Madrid: Tecnos.

Mitcham, C. (1989). ¿Qué es la filosofía de la tecnología? Barcelona: Editorial Antrophos.

Moravec, H. (1988). El hombre mecánico. El futuro de la robótica y la inteligencia humana. Madrid: Ediciones Temas de Hoy. 
Müller, V. C., \& Bostrom, N. (2014). Future progress in artificial intelligence: A Survey of Expert Opinion. En V. C. Müller (Ed.), Fundamental Issues of Artificial Intelligence (pp. 555-572). Berlín: Springer.

Ortega y Gasset, J. (1965a). El mito del hombre allende la técnica. En J. Ortega y Gasset, Obras completas (pp. 617-624), tomo ix. Madrid: Revista de Occidente.

Ortega y Gasset, J. (1965b). Meditación de la técnica. Madrid: Editorial Espasa-Calpe.

Ortega y Gasset, J. (2008). La rebelión de las masas. Madrid: Tecnos.

Rouhiainen, L. (2018). Inteligencia artificial. 101 cosas que debes saber hoy sobre nuestro futuro. Barcelona: Editorial Planeta.

Schwab, K. (2016). La cuarta revolución industrial. Barcelona: Debate.

Tegmark, M. (2017). Life 3.0: being human in the age of Artificial Intelligence. Great Britain: Penguin Random House.

Turing, A. M. (1950). Computing Machinery and Intelligence. Mind, 49, 433-460. DoI: https://doi.org/10.1093/mind/LIX.236.433.

Walsh, T. (2017). Android Dreams: The Past, Present and Future of Artificial Intelligence. London: C Hurst \& Co Publishers Ltd. 